\title{
Strategies for Dealing with Problems in Robotised Unscrewing Operations
}

\author{
Jun Huang ${ }^{(凶)}(\mathbb{D}$, Duc Truong Pham (D), Ruiya Li (D), Kaiwen Jiang (D), Dalong Lyu, \\ and Chunqian Ji (iD \\ Department of Mechanical Engineering, School of Engineering, University of Birmingham, \\ Birmingham B15 2TT, UK \\ \{j.huang.1,d.t.pham, C.Ji\}@bham.ac.uk, \{rx1721,xj797, \\ dxl887\} @student. bham.ac.uk
}

\begin{abstract}
Disassembly is the first step in a remanufacturing process chain with unscrewing being usually the most frequent task. In previously reported research in the authors' laboratory, a new method has been developed for using robots to unfasten screws. Uncertainties and variability in the physical condition of screws induced by dirt, rust, or mechanical damage pose difficulties for such robotised unscrewing systems. There are three common failure modes: screwdriver missing screw head, screwdriver slipping on screw head and screw too tight to remove. This paper presents strategies to handle these failure modes, making the developed robotised method more robust and reliable. The strategies include conducting a second search and second unfastening trial as well as involving collaboration with a human operator. Tests were carried out to validate the proposed strategies. The results show that the strategies could deal with the failure modes, enabling $100 \%$ successful operation.
\end{abstract}

Keywords: Remanufacturing $\cdot$ Robotic disassembly $\cdot$ Automated unscrewing

\section{Introduction}

Remanufacturing is an increasingly significant part of a circular economy, delivering economic, environmental and social benefits [1, 2]. In remanufacturing, disassembly of end-of-life (EoL) products is a critical first step [3]. Disassembly is labour intensive and skill intensive and disassembly costs usually represent a substantial proportion of the cost of the remanufactured product. Robotic technology has been proposed for disassembly $[4,5]$, with a range of approaches having been explored for robotic disassembly applications [6-8]. For example, robot-assisted disassembly was used for dismantling electronic products as early as 1994 [9]. Robots have many advantages including high accuracy, speed and repeatability and the ability to handle repetitive and tedious disassembly operations such as unscrewing [10]. Screws are very common mechanical fasteners as they can be dismantled non-destructively for future maintenance, repair, remanufacturing and recycling [11]. However, unscrewing is difficult to be automated. This is due to uncertainties and variability in the location and the physical condition of

(C) The Author(s) 2021

S. Ratchev (Ed.): IPAS 2020, IFIP AICT 620, pp. 93-107, 2021.

https://doi.org/10.1007/978-3-030-72632-4_7 
screws in EoL products caused by corrosion, contaminants (grease and dirt), deformation and other forms of mechanical damage [12].

A robotic system was prototyped for automated screw removal from e-waste products such as laptops [13]. Computer vision and force sensing were employed to locate screws automatically. An accelerometer mounted on a screwdriver was used to detect when a screw had been completely detached from a mating threaded hole. A screw removal success rate of $96.5 \%$ was achieved, which was affected by the lighting conditions of the computer vision system. A robotic workstation fitted with Kinect cameras was developed for autonomous disassembly of electric vehicle motors [14]. A detection algorithm was proposed to locate the position and determine the type of the screws to be removed by the robot. A hybrid workstation with a collaborative robot was presented for performing unscrewing in EoL electric vehicles (EV) batteries which contained substances hazardous to human health $[15,16]$. A bit holder was designed to allow the robot to change different socket wrench bits. An electric screwdriver and a camerabased location detection system were also proposed for automatic unscrewing tasks [17]. A spiral search strategy was designed for a robot to locate and engage a screw head, ensuring that no position was scanned twice [18]. In addition, a robotised screwing and unscrewing technique was developed for dismantling electronic devices [19]. A robot was released by Apple in 2016 for removing screws on EoL iPhones to disassemble them for remanufacturing and recycling to reduce e-waste [20].

The above research has mainly focused on methods and devices for automated unscrewing. Few studies have considered the uncertain physical conditions of screws in EoL products. Four common conditions have been identified in automated unscrewing operations [21]. They include three failure modes: (1) screwdriver missing screw head, (2) screwdriver slipping on screw head and (3) screw too tight to remove. In previously reported research in the authors' laboratory, a new method was developed for using a collaborative robot to unfasten screws [22]. This paper presents strategies for handling the above failure modes in robotised unscrewing operations, making the developed method more robust and reliable.

The remainder of this paper is organised as follows. Section 2 briefly introduces a human-robot collaborative disassembly cell built in the authors' laboratory and the automated unscrewing method implemented in that cell. Section 3 describes the proposed failure handling strategies. Section 4 reports on tests carried out to validate the proposed strategies. Section 5 concludes the paper and provides suggestions for future work.

\section{Automated Unscrewing Method}

\subsection{A Human-Robot Collaborative Disassembly Cell}

Figure 1 shows a human-robot collaborative disassembly cell for unfastening screws built in the authors' laboratory. The cell mainly composes a collaborative robot (KUKA LBR iiwa 14 R800), an electrical nutrunner (torque spindle, Georges Renault SAS MC51-10) and a geared offset attachment (LUBBERING basic line 4-22-15). The nutrunner was installed on the flange of robot. A spindle controller (Georges Renault SAS, 8216-CVIC $\mathrm{H}-4)$ was used to control and programme the nutrunner. The geared offset attachment 
mounted on the nutrunner could unfasten Hexagonal head bolts with flanges, as shown in Fig. 1. When connected, the robot could trigger and stop the nutrunner.

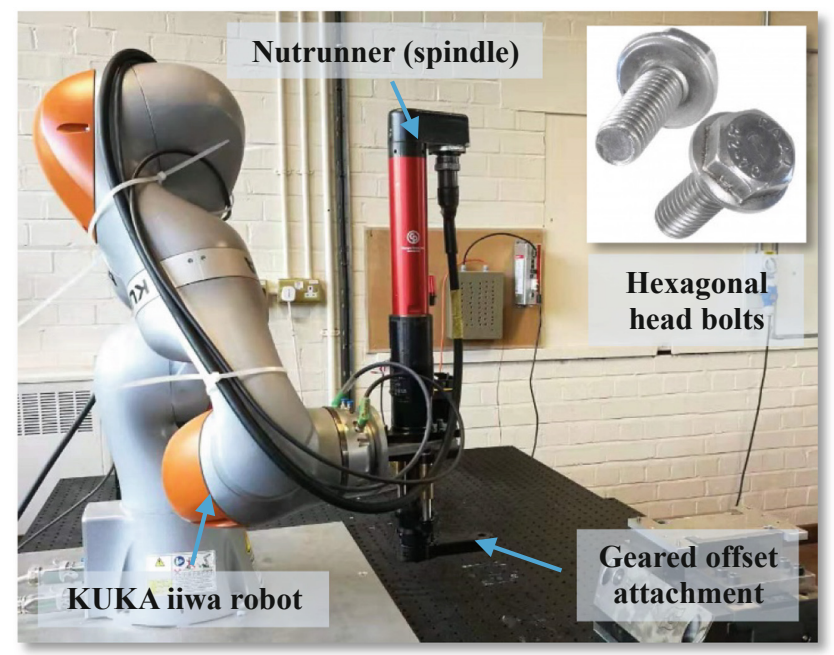

Fig. 1. A robotic cell for automated unscrewing.

The robot has active compliance control using a Cartesian impedance controller. This facilitates human-robot collaboration for complex disassembly operations in the cell. A human operator can work alongside the robot directly without safety guarding, providing the cell with higher flexibility and adaptability and thus a better ability to handle disassembly uncertainties.

\subsection{Automated Unscrewing Process}

Figure 2 shows the developed automated unscrewing process, which involves four stages: approach, search and engage, unfasten, and assess and leave. The control strategies of torque, position and active compliance are adopted during the process. The corresponding control method of each stage is briefly introduced below. More details about the automated unscrewing method could be found in [22].

1) Approach. The robot with a nutrunner moves to an estimated position above screw head. The nutrunner rotates in the fastening direction at a low speed to ensure that any loose screw will be tightened up before unfastening, making the following stage easier to control. Then, the robot turns on its compliant mode. The compliant behaviour of the robot is achieved by impedance control, which is modelled on a virtual spring-damper system with configurable stiffness and damping [23]. Thanks to compliance, the robot can avoid hard collisions with humans operating in its workspace.

2) Search and engage. Due to factors such as the uncertain mechanical condition of screws and the limited accuracy of robot motion, there is a position error between 


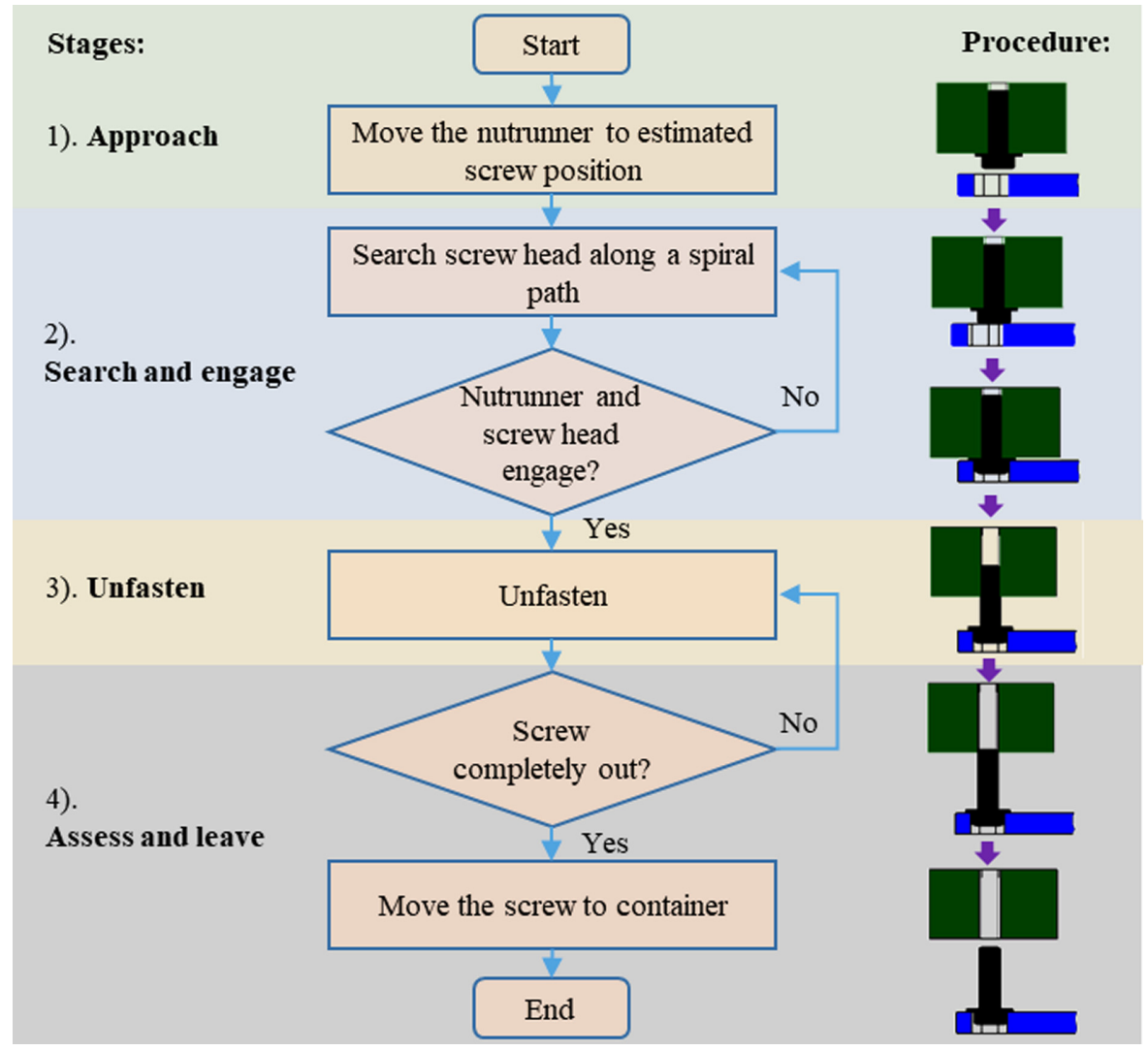

Fig. 2. Automated unscrewing process.

the hexagonal hole in the geared offset attachment and the hexagonal screw head. A spiral motion is designed for the robot to search for and engage the screw head. The equations of the spiral path employed are [22]:

$$
\left\{\begin{array}{l}
x=\frac{f_{s}}{\pi} \sqrt{\frac{2 \pi n_{s}}{5} t} \cos \left(\sqrt{\frac{2 \pi n_{s}}{5} t}\right) \\
y=\frac{f_{s}}{\pi} \sqrt{\frac{2 \pi n_{s}}{5} t} \sin \left(\sqrt{\frac{2 \pi n_{s}}{5} t}\right)
\end{array}\right.
$$

where $x$ and $y$ are the coordinates along the $\mathrm{X}$ and $\mathrm{Y}$ axes, respectively. $f_{S}$ is the size of the hexagonal screw head chamfer. $n_{s}$ is the rotating speed of the tool and $t$ is search time.

3) Unfasten. Once the tool and screw head have engaged, the robot stops its search motion and the nutrunner rotates in the unfastening direction. Under the compliant mode, the robot can follow the movement of the screw during run-out.

4) Assess and leave. When the screw has been separated from the screw hole, the screw oscillates slightly along its axis due to the chamfer at the end of the screw threads and continuous rotation of the tool. The position information of the robot along the 
direction of the screw axis is employed to determine when the screw is completely out. Finally, the separated screw is moved away.

\section{Mitigating Strategies for Failure Modes}

\subsection{Strategies for Dealing with Failure Modes}

Three common failure modes are identified in automated unscrewing operations, which are: (1) screwdriver missing screw head, (2) screwdriver slipping on screw head and (3) screw too tight to remove [21]. Figure 3 illustrates the flow chat of the proposed strategies to deal with these problems.

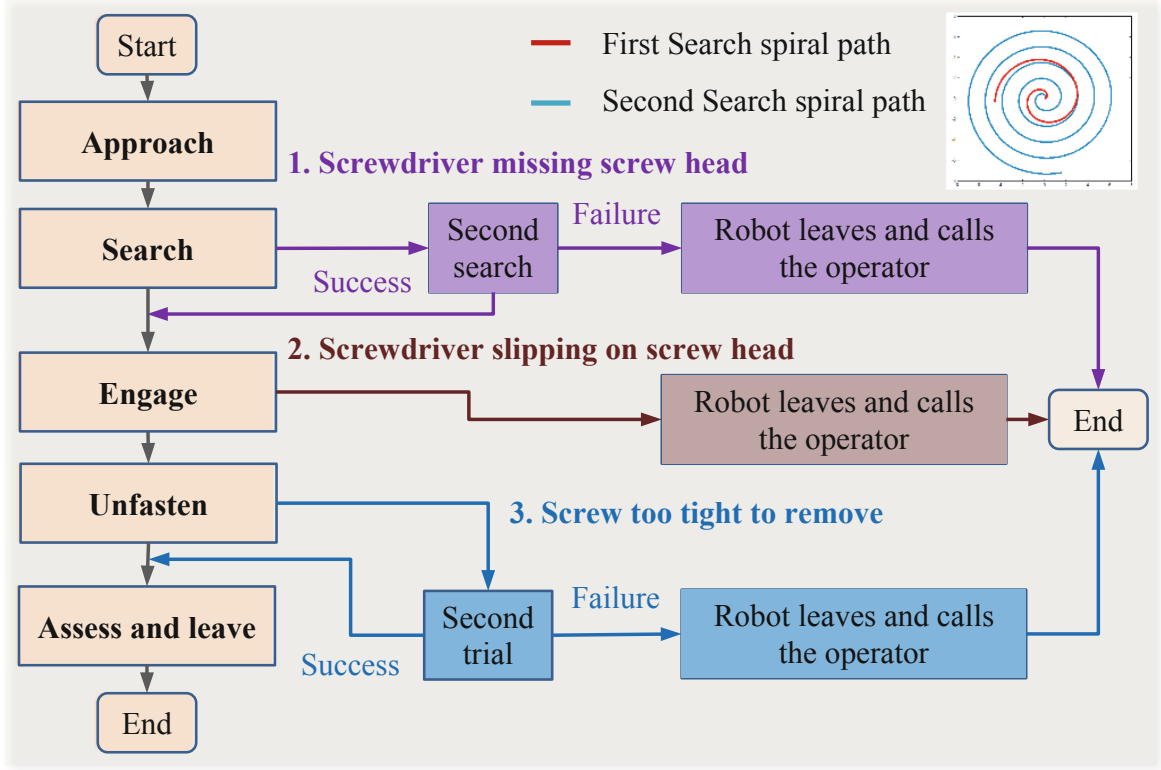

Fig. 3. The flow chart of the proposed strategies.

If the position error were without the spiral search range, the tool would miss the screw head in its search motion (failure mode 1). Conducting a second spiral search along a path with a larger range and higher density is proposed as a strategy to handle this problem, as shown in Fig. 3. If the second search succeeds, subsequent unscrewing steps will proceed. Otherwise, human-robot collaboration will be implemented to cope with this screw in the human-robot collaborative disassembly cell. The robot will leave its search position and call a human operator to come to dismantle the screw.

Due to rust, corrosion or mechanical damage to a screw, the tool may slip on the screw head (failure mode 2). It is difficult for the robot to disassemble screws in such a situation. Once the situation is detected by the robot, the operator will again be called to handle it. If a screw is too tightly fixed, the tool is unable to disassemble it because the 
required loosening torque exceeds the maximum capability of the tool (failure mode 3). Although the screw has not been successfully removed in the unfastening operation, the impact force exerted by the tool might have loosened it. A second unfastening attempt is made to apply another impact force to try and release the screw. If this fails again, the robot will leave the current position and call the operator.

\subsection{Detection Methods for Failure Modes}

Before adopting the above strategies, it is critical for the robot to detect these failure modes during automated unscrewing. Information regarding the position and torque of the robot is employed for this detection. Figure 4 shows how information on the position of the robot tool is used to determine a search failure. Once the tool fits in the screw head, its position along the $\mathrm{Z}$ axis will increase from $P_{z 0}$ to $P_{z 1}$. The following equation is used to determine if engagement has occurred:

$$
P_{z 1}-P_{z 0}>\Delta P
$$

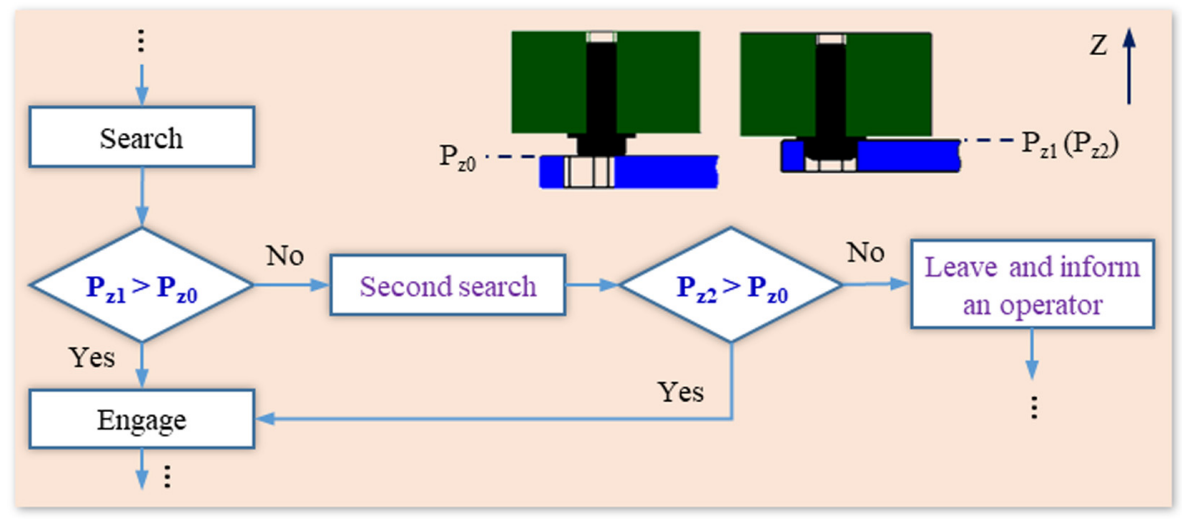

Fig. 4. Detection of failure mode 1 .

where $P_{z 0}$ and $P_{z 1}$ are the initial and end positions of the tool during search along the $\mathrm{Z}$ axis, respectively. $\Delta P$ is a set threshold value for position change and is related to the size of the screw head. In the second search motion, the same way could be employed to determine whether the tool fits in the screw head or not, as shown in Fig. 4. 
Robot's torque information is employed to detect failure modes 2 and 3, as shown in Fig. 5. A target torque value $\left(T_{t 0}\right)$ is set to stop the search, once the tool engages the screw head during a searching process. As shown in Eq. (3), the applied torque on the tool $\left(T_{e}\right)$ will increase once the tool engages the screw head due to its slow rotation in fastening direction. However, if the tool slips on screw head, torque on the tool could not increase to the target value.

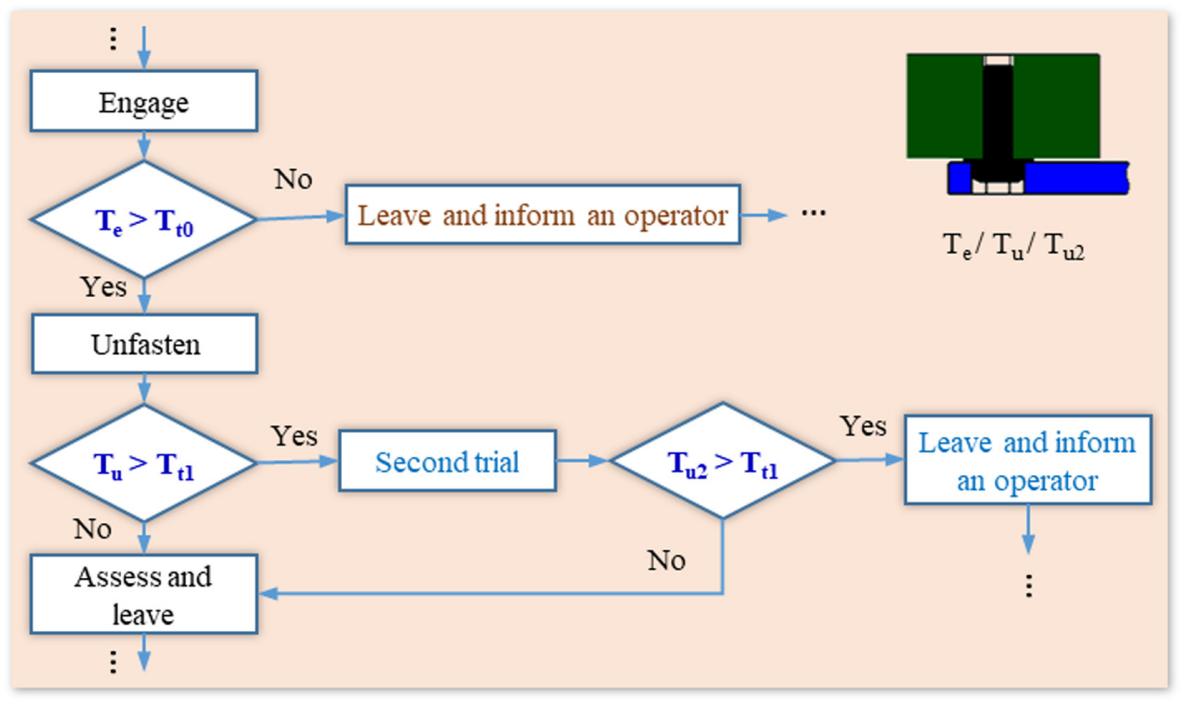

Fig. 5. Detections of failure modes 2 and 3.

$$
T_{e}>T_{t 0}
$$

If the screw is too tight to be removed, the applied torque on robot tool $\left(T_{u}\right)$ will increase and exceed the maximum safe torque value $\left(T_{t 1}\right)$, as expressed in Eq. (4). Therefore, the torque on robot tool during unfastening process could be used to detect the failure mode 3 .

$$
T_{u}>T_{t 1}
$$

\section{Experimental Tests and Results}

The robot cell in Fig. 1 was implemented for experimental tests to validate the above strategies. Hexagon headed screws $(\mathrm{M} 6 \times 16 \mathrm{~mm})$ with flange were installed on a metal plate. Table 1 lists the set speed and torque values of the nutrunner in stages 2 and 3. A flashing LED strobe light connected to robot controller was adopted to inform a human operator to handle the screws when the robot was unable to do that. 
Table 1. Parameters of the nutrunner.

\begin{tabular}{l|l|l|l}
\hline \multicolumn{2}{l|}{ Search and engage } & Unfasten \\
\hline Rotating speed of the tool & $25 \mathrm{r} / \mathrm{min}$ & Rotating speed of the tool & $100 \mathrm{r} / \mathrm{min}$ \\
\hline Target fastening torque & $14 \mathrm{Nm}$ & Maximum unfastening torque & $25 \mathrm{Nm}$ \\
\hline
\end{tabular}

\subsection{Tests and Results for Dealing with Failure Mode 1}

The proposed strategy of second search worked and successfully engaged the screw head after the first search failed. Figure 6 presents selected image frames captured from the testing process. Figure 6(a) shows the robot with the nutrunner approaching an estimated position of the screw head. The failure of the first search can be seen in Fig. 6(b). Figure 6(c) shows that the tool capturing and engaging the screw head within the second spiral search. The unfastening of the screw is shown in Fig. 6(d). Once the unfastening stage finished, the screw was taken away by the robot, as shown in Fig. 6(e).
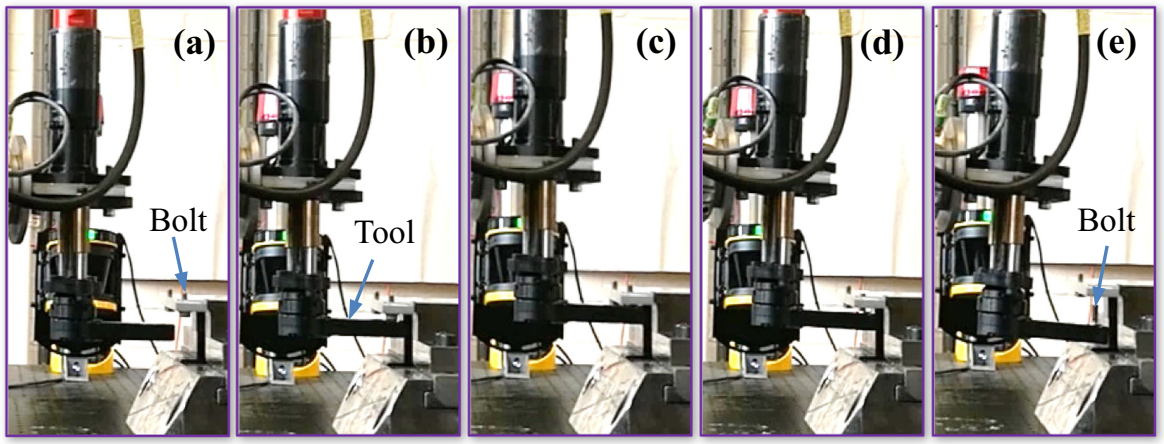

Fig. 6. Strategy of conducting a second search: (a) approach, (b) the first search fails, (c) the second search succeeds, (d) unfasten, and (e) assess and leave.

Figure 7 shows the torque and position information measured by the robot during the unscrewing process. Four stages are illustrated clearly. The torque value of $-2.5 \mathrm{Nm}$ was set as a threshold value to stop the search once the tool engaged the screw head. Position information was used to determine whether the first search was successful and when the screw was completely out during unfastening. 


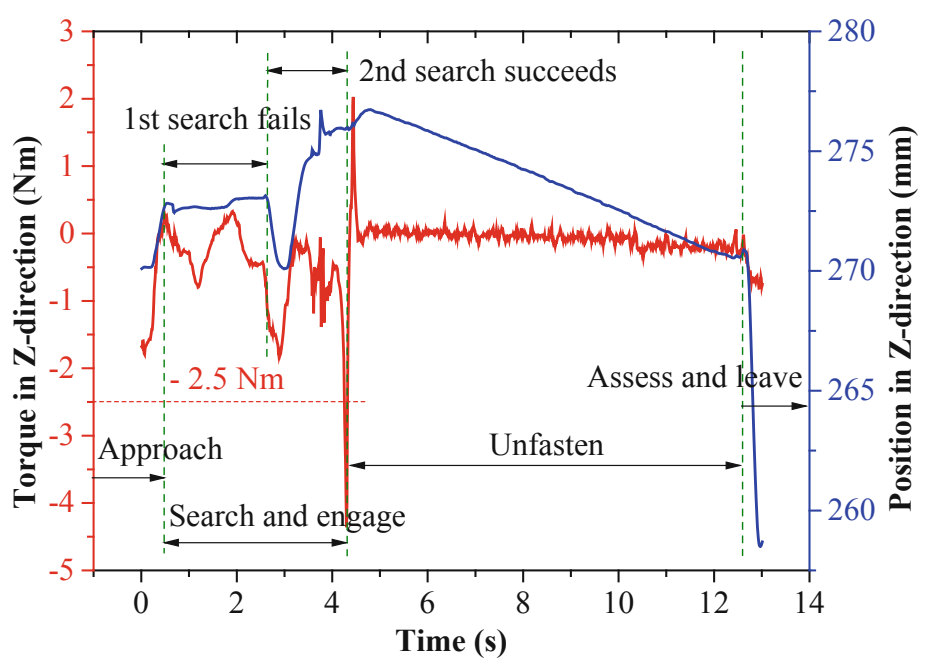

Fig. 7. Torque and position information recorded by the robot.

The strategy for dealing with the failure of both searches was tested. Figure 8 illustrates the selected image frames captured from the unscrewing process. After the tool approached the screw head (Fig. 8(a)), both the first and second searches failed, as shown in Fig. 8(b) and (c), respectively. Then, the robot left its current position and turned on the strobe light to inform an operator (Fig. 8(d)). Figure 8(e) shows the operator unfastening the screw with a spanner. Figure 9 shows the measured torque and position information by the robot during the unscrewing process. The maximum torque of the robot did not reach the threshold value of -2.5 or $2.5 \mathrm{Nm}$, which was used to stop the search once the tool engaged with the screw head.
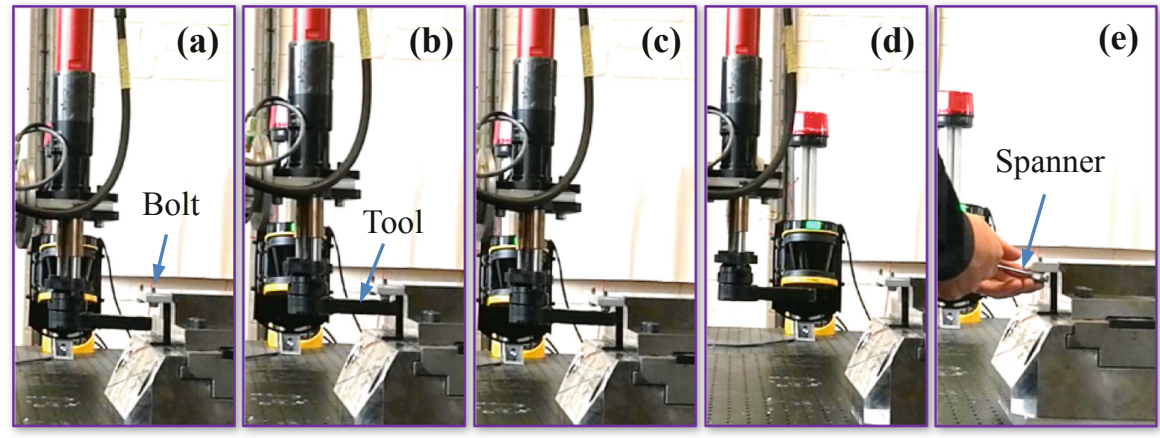

Fig. 8. Strategy for dealing with the failure of both searches, (a) approach, (b) the first search fails, (c) the second search fails, (d) leave and inform an operator, and (e) handle the screw by the operator. 


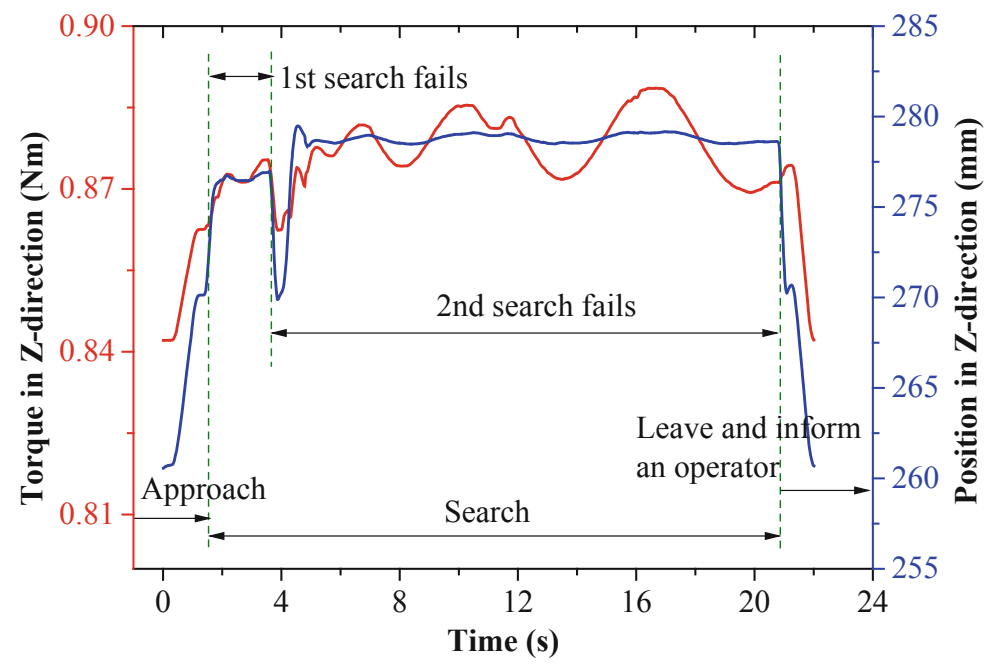

Fig. 9. Torque and position information recorded by the robot.

\subsection{Tests and Results for Dealing with Failure Mode 2}

Figure 10 shows selected image frames captured from the testing of the strategy proposed for handling the failure mode associated with the tool slipping on the screw head. Figure 10(c) shows the tool slipped on the head of screw after the nutrunner approached the screw (Fig. 10(a)) and fitted in the screw head (Fig. 10(b)). Once slipping was detected, the robot left its current position and called the operator using the strobe light (Fig. 10(d)) to handle the screw with a special tool (Fig. 10(e)). Figure 11 depicts the recorded position and torque information by the robot. During slipping, the tool stayed nearly in a fixed position with small fluctuations.
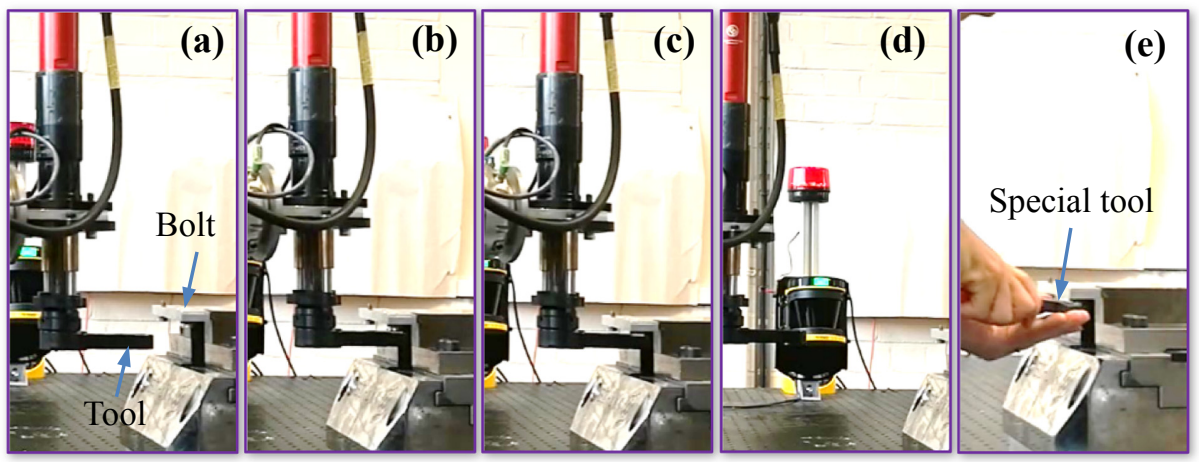

Fig. 10. Strategy for dealing with slipping, (a) approach, (b) search and fit in, (c) slip, (d) leave and inform an operator, and (e) handle the screw by the operator. 


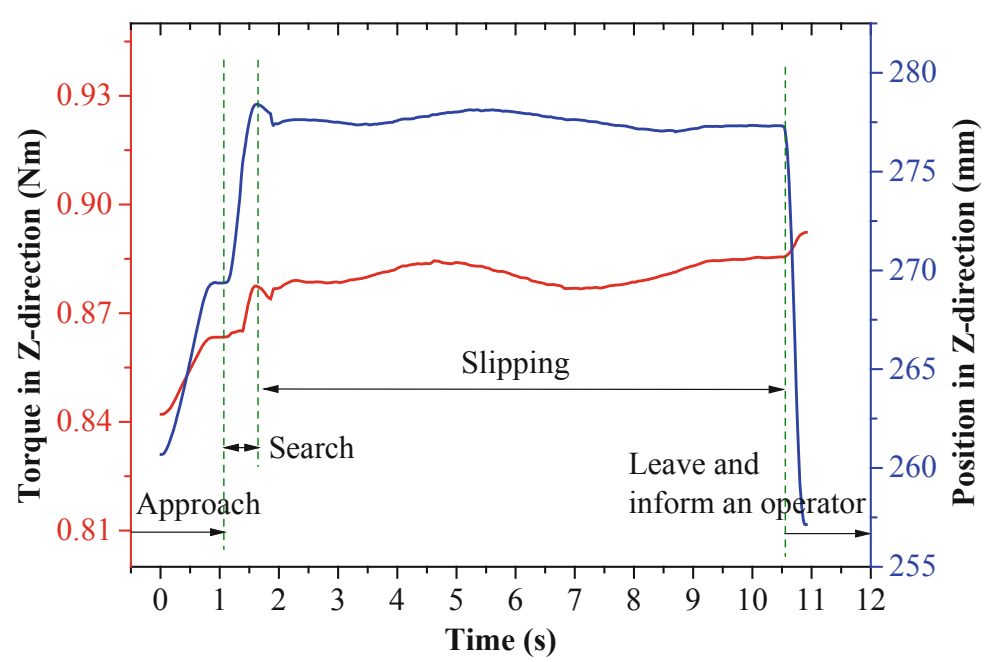

Fig. 11. Torque and position information recorded by the robot.

\subsection{Tests and Results for Dealing with Failure Mode 3}

Figure 12 shows image frames captured in the test of the strategy to handle a screw that is too tight to remove. Figure 12(a) depicts the tool approaching the screw head. Then, the tool searched for and engaged with the screw head, as shown in Fig. 12(b). However, the tool was unable to unfasten the screw because it was too tight (Fig. 12(c)). The tool left its current position and turned on the strobe light (Fig. 12(d)). Finally, an operator came to disassemble the screw with a hammer and a spanner (Fig. 12(e)). Figure 13 shows the recorded torque and position of the robot. Before the robot left, the position of the tool almost did not change after engaging with the screw head.

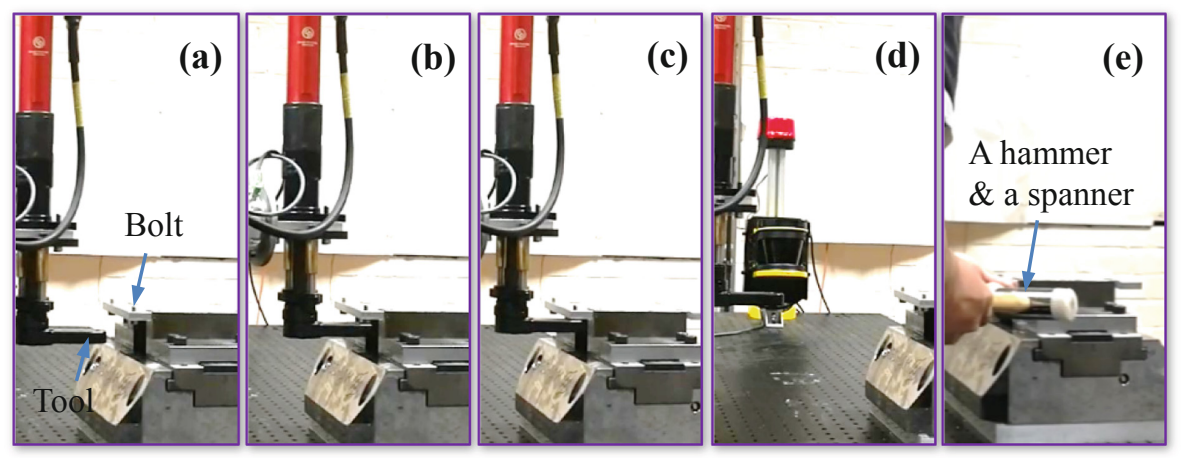

Fig. 12. Strategy for dealing with a screw that is too tight to remove, (a) approach, (b) search and engage, (c) the screw too tight to remove, (d) leave and inform an operator, and (e) handle the screw by the operator. 


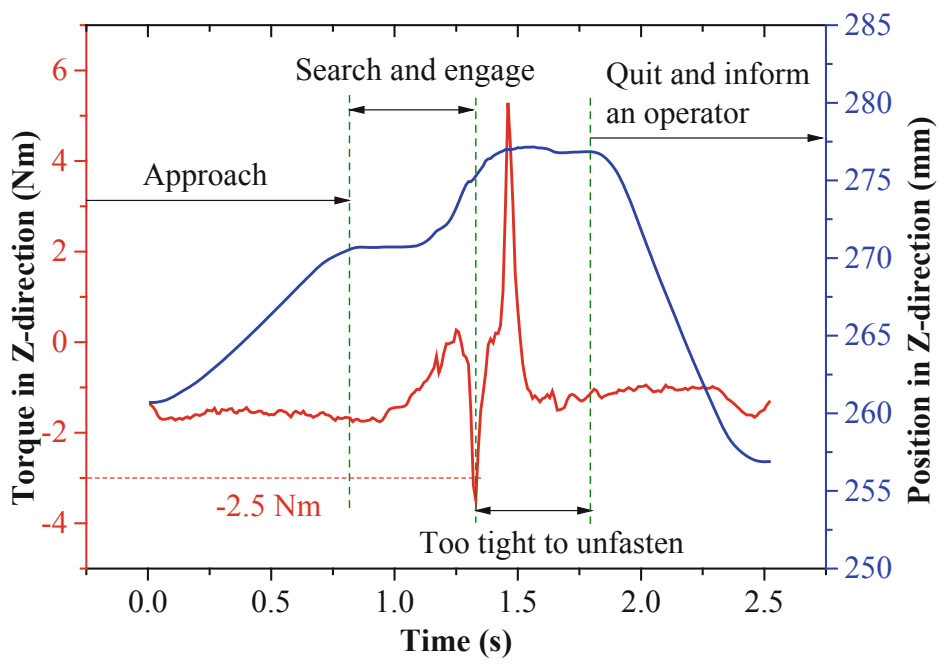

Fig. 13. Torque and position information recorded by the robot.

The proposed strategy involving a second unfastening attempt was tested. Figure 14 illustrates the selected image frames captured during the test. After approaching the screw (Fig. 14(a)), the robot searched for and engaged with the screw head (Fig. 14 (b)). Figure 14(c) shows the first unfastening failed because the screw was too tight. The second unfastening trial succeeded as shown in Fig. 14(d). After the screw was separated from the screw hole, the robot moved the screw away (Fig. 14(e)). The large torque fluctuations during the two unfastening trials are clearly captured, as shown in Fig. 15.

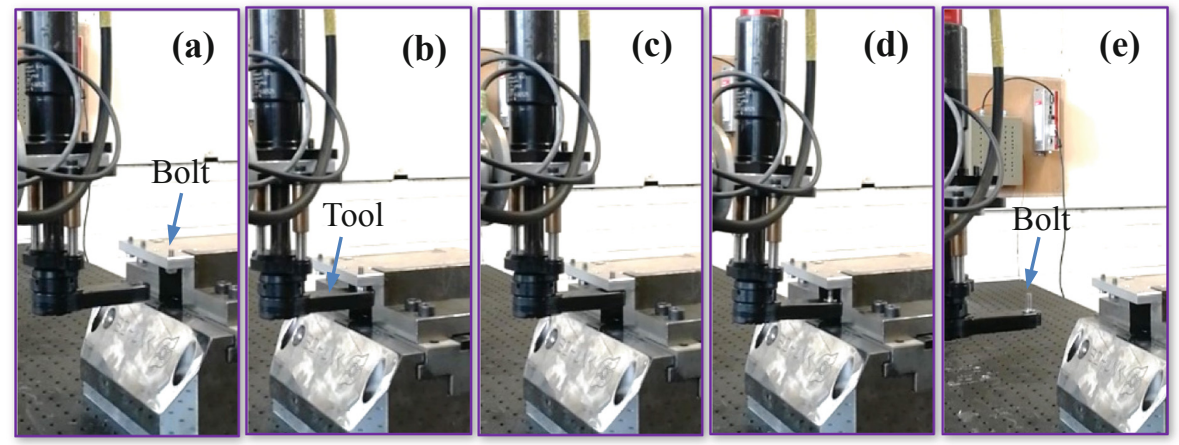

Fig. 14. Strategy of conducting a second unfastening attempt, (a) approach, (b) search and engage, (c) the first attempt fails, (d) the second attempt succeeds, and (e) assess and leave. 


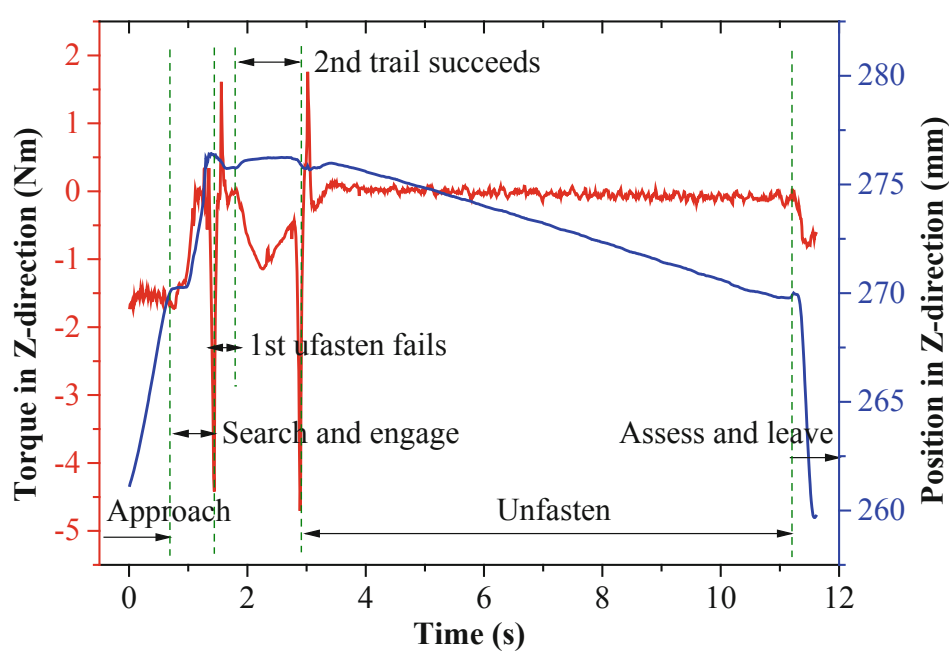

Fig. 15. Torque and position information recorded by the robot.

\section{Conclusion}

Unscrewing is one of the most common disassembly operations. Automating unscrewing can release people from tedious and repetitive work. Unlike the case of screwing in assembling a new product, unscrewing is difficult to automate due to the poor physical conditions of screws in EoL products. This paper has presented strategies for handling the three main failure modes in automated unscrewing operations, namely, screwdriver missing screw head, screwdriver slipping on screw head and screw too tight to remove. The strategies involve conducting a second search and second unfastening trial or collaborating with a human operator. The paper has briefly introduced a robotic unscrewing method implemented in a human-robot collaborative disassembly cell. The unscrewing process has four stages: approach, search and engage, unfasten, and assess and leave. Experiments have been conducted to test and validate the proposed strategies. The results obtained have demonstrated the feasibility of the strategies which can be adopted to enhance the robustness and reliability of the robotised unscrewing method developed.

In the future, the proposed strategies will be integrated and further tests will be conducted to improve the robustness and efficiency of the robotised unscrewing method. The unscrewing system will also be adapted to make it suitable for different types of screws and bolts.

Acknowledgments. This research was supported by the Engineering and Physical Sciences Research Council (EPSRC) (Grant. No. EP/N018524/1). 


\section{Appendix}

The automated unscrewing method presented in Sect. 2 was implemented in a turbocharger disassembly demonstration which can be viewed on YouTube at https://www. youtube.com/watch?v=kOwGe_LbLzs.

\section{References}

1. Kerin, M., Pham, D.T.: A review of emerging industry 4.0 technologies in remanufacturing. J. Cleaner Prod. 237, 117805 (2019)

2. Stahel, W.R.: The circular economy. Nat. News 531(7595), 435 (2016)

3. Vongbunyong, S., Chen, W.H.: Disassembly Automation. Disassembly Automation. SPLCEM, pp. 25-54. Springer, Cham (2015). https://doi.org/10.1007/978-3-319-15183-0_3

4. AUTOREMAN project. http://autoreman.altervista.org/index.html. Accessed 03 Oct 2019

5. Huang, J., Pham, D.T., Wang, Y., Ji, C., Xu, W., Liu, Q.: A strategy for human-robot collaboration in taking products apart for remanufacture. FME Trans. 47, 731-738 (2019)

6. Li, J., Barwood, M., Rahimifard, S.: Robotic disassembly for increased recovery of strategically important materials from electrical vehicles. Robot. Comput. Integr. Manuf. 50, 203-212 (2018)

7. Palmieri, G., Marconi, M., Corinaldi, D., Germani, M., Callegari, M.: Automated disassembly of electronic components: feasibility and technical implementation. In: ASME 2018 International Design Engineering Technical Conferences and Computers and Information in Engineering Conference. American Society of Mechanical Engineers (2018)

8. Kristensen, C.B., Sørensen, F.A., Nielsen, H.B.D.B., Andersen, M.S., Bendtsen, S.P., Bøgh, S.: Towards a robot simulation framework for E-waste disassembly using reinforcement learning. In: 29th International Conference on Flexible Automation and Intelligent Manufacturing (2019)

9. Weigl, A.: Requirements for robot assisted disassembly of not appropriately designed electronic products: lessons from first studies. In: Proceedings of 1994 IEEE International Symposium on Electronics and the Environment, pp. 337-342 (1994)

10. Huang, J., et al.: A screw unfastening method for robotic disassembly. In: the International Conference on Sustainable Smart Manufacturing (S2M 2019) (2019)

11. Jia, Z., Bhatia, A., Aronson, R.M., Bourne, D., Mason, M.T.: A survey of automated threaded fastening. IEEE Trans. Autom. Sci. Eng. 16(1), 298-310 (2019)

12. Gao, Y., Feng, Y., Wang, Q., Zheng, H., Tan, J.: A multi-objective decision making approach for dealing with uncertainty in EOL product recovery. J. Cleaner Prod. 204, 712-725 (2018)

13. DiFilippo, N.M., Jouaneh, M.K.: A system combining force and vision sensing for automated screw removal on laptops. IEEE Trans. Autom. Sci. Eng. 15(2), 887-895 (2018)

14. Bdiwi, M., Rashid, A., Putz, M.: Autonomous disassembly of electric vehicle motors based on robot cognition. In: Proceedings of the IEEE International Conference on Robotics and Automation (ICRA 2016), pp. 2500-2505 (2016)

15. Chen, W.H., Wegener, K., Dietrich, F.: A robot assistant for unscrewing in hybrid humanrobot disassembly. In: Proceedings of the IEEE International Conference on Robotics and Biomimetics (ROBIO 2014), pp. 536-541 (2014)

16. Gerbers, R., Wegener, K., Dietrich, F., Dröder, K.: Safe, flexible and productive humanrobot-collaboration for disassembly of lithium-ion batteries. In: Kwade, A., Diekmann, J. (eds.) Recycling of Lithium-Ion Batteries. SPLCEM, pp. 99-126. Springer, Cham (2018). https://doi.org/10.1007/978-3-319-70572-9_6 
17. Wegener, K., Chen, W.H., Dietrich, F., Dröder, K., Kara, S.: Robot assisted disassembly for the recycling of electric vehicle batteries. Procedia CIRP 29, 716-721 (2015)

18. Gerbers, R., Mücke, M., Dietrich, F., Dröder, K.: Simplifying robot tools by taking advantage of sensor integration in human collaboration robots. Procedia CIRP 44, 287-292 (2016)

19. Mironov, D., Altamirano, M., Zabihifar, H., Liviniuk, A., Liviniuk, V., Tsetserukou, D.: Haptics of screwing and unscrewing for its application in smart factories for disassembly. Haptics Sci. Technol. Appl. 10894, 428-439 (2018)

20. How and why Apple's robot Liam disassembles iPhones. https://www.zdnet.com/article/howand-why-apples-robot-liam-disassembles-iphones/. Accessed 2019

21. Apley, D.W., Seliger, G., Voit, L., Shi, J.: Diagnostics in disassembly unscrewing operations. Int. J. Flex. Manuf. Syst. 10(2), 111-128 (1998)

22. Li, R., et al.: Unfastening of hexagonal headed screws by a collaborative robot. IEEE Trans. Autom. Sci. Eng. 17, 1-14 (2020)

23. KUKA Sunrise.OS 1.11. http://www.oir.caltech.edu/twiki_oir/pub/Palomar/ZTF/KUKARo boticArmMaterial/KUKA_SunriseOS_111_SI_en.pdf. Accessed 03 Oct 2019

Open Access This chapter is licensed under the terms of the Creative Commons Attribution 4.0 International License (http://creativecommons.org/licenses/by/4.0/), which permits use, sharing, adaptation, distribution and reproduction in any medium or format, as long as you give appropriate credit to the original author(s) and the source, provide a link to the Creative Commons license and indicate if changes were made.

The images or other third party material in this chapter are included in the chapter's Creative Commons license, unless indicated otherwise in a credit line to the material. If material is not included in the chapter's Creative Commons license and your intended use is not permitted by statutory regulation or exceeds the permitted use, you will need to obtain permission directly from the copyright holder.

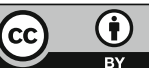

